Supporting information for

\title{
Aging of Volatile Organic Compounds in October 2017 Northern California Wildfire Plumes
}

Yutong Liang ${ }^{1 *}$, Robert J. Weber ${ }^{1}$, Pawel K. Misztal ${ }^{1,2}$, Coty N. Jen ${ }^{1,3}$, Allen H. Goldstein ${ }^{1,4^{*}}$

${ }^{1}$ Department of Environmental Science, Policy, and Management, University of California, Berkeley, Berkeley, CA, 94720, USA

${ }^{2}$ Department of Civil, Architectural and Environmental Engineering, University of Texas at Austin, Austin, TX 78712, USA

${ }^{3}$ Department of Chemical Engineering, Carnegie Mellon University, Pittsburgh, PA 15213, USA

${ }^{4}$ Department of Civil and Environmental Engineering, University of California, Berkeley, California 94720, USA

Supplement includes:

Supplementary text

Figures S1-S10

Table S1 


\section{SUPPLEMENTARY TEXT}

\section{Chemical plume age estimation}

We estimated the chemical ages of the plumes by using four primary BB VOC species: furan, furfural, guaiacol and acetonitrile. The first three compounds have higher reaction rate constants with $\mathrm{OH}$ (on the order of $10^{-11} \mathrm{~cm}^{-3}$ molecule ${ }^{-1}$ ), while acetonitrile has an almost negligible reaction rate constant with $\mathrm{OH}$ (on the order of $10^{-14} \mathrm{~cm}^{-3}$ molecule ${ }^{-1}$ ) with respect to the relevant plume transport times. ${ }^{1}$ We paired furan, furfural and guaiacol with acetonitrile to calculate the plume age, using the approach by Kleinman et al. ${ }^{2}$ The plume age $\left(t_{\text {age }}\right)$ can be calculated by:

$$
t_{\text {age }}=\frac{\ln \left(\frac{\left[X_{i}\right]}{\left[X_{i}\right]_{0}}\right)-\ln \left(\frac{\left[\mathrm{CH}_{3} \mathrm{CN}\right]}{\left[\mathrm{CH}_{3} \mathrm{CN}\right]_{0}}\right)}{\left(k_{\mathrm{CH}_{3} \mathrm{CN}}-k_{i}\right)[\mathrm{OH}]}=\frac{\ln \left(\frac{\left[X_{i}\right]}{\left[\mathrm{CH}_{3} \mathrm{CN}\right]}\right)-\ln \left(\frac{\left[X_{i}\right]_{0}}{\left[\mathrm{CH}_{3} \mathrm{CN}\right]_{0}}\right)}{\left(k_{\mathrm{CH}_{3} \mathrm{CN}}-k_{i}\right)[\mathrm{OH}]}
$$

where $\left[\mathrm{X}_{\mathrm{i}}\right]$ is the concentration of furan, furfural or guaiacol, $\left[\mathrm{CH}_{3} \mathrm{CN}\right]$ is the concentration of acetonitrile. The kinetic reaction rate constants, $k$, are for reactions with $\mathrm{OH} .\left[\mathrm{X}_{\mathrm{i}}\right]_{0}$ and $\left[\mathrm{CH}_{3} \mathrm{CN}\right]_{0}$ are the initial concentrations of the BB VOCs when they were emitted. As we did not have measurements at the points of emission, we instead used the average $\left[\mathrm{X}_{\mathrm{i}}\right] /\left[\mathrm{CH}_{3} \mathrm{CN}\right]$ emission ratios of relevant fuels reported in Koss et al. ${ }^{3}$, i.e., 1.9, 2.1 and $1.3 \mathrm{ppb} / \mathrm{ppb}$ acetonitrile for furan, furfural and guaiacol, respectively. In addition, we estimated the plume age by assuming atmospheric oxidant level equivalent to $[\mathrm{OH}]=1.5 \times 10^{6}$ molecules $\mathrm{cm}^{-3}$. Although the initial concentrations have high uncertainties because the emission factors of these compounds from the Napa/Sonoma County fires were not directly measured, the ages of the plumes estimated by the three pairs differ by only $19 \%$ in terms of normalized root mean square error. From S1, changing the initial concentration ratio will only change the constant term of $t_{\text {age. }}$ We averaged the plume ages 
measured by the three pairs of compounds to calculate the chemical plume age to be used for subsequent analyses. It is worth noting that in previous studies, acetonitrile was observed to show transient urban peaks, ${ }^{4,5}$ which might come from nearby chemical laboratories using acetonitrile as solvent, as suggested by Coggon et al. ${ }^{4}$ and Huangfu et al..$^{5}$ and We did not find such peaks in the dense plume periods on Oct 10-13 and conclude that any urban emissions were minor compared to the wildfire emissions at those times. However, such transient peaks clearly appeared in the daytime on Oct 16, and Oct 18-20. For consistency, like Coggon et al. ${ }^{4}$, we applied a lowpass filter (60-minute moving median) to preprocess the acetonitrile data. We compared the chemical plume ages with physical plume ages estimated from backward trajectory analysis (Section 2.3). For the fresh plumes with less than 3-4 hours physical age, the chemical plume ages had good agreement with the physical plume ages.

\section{Backward trajectory calculation and Concentration-Weighted-Trajectory (CWT) analysis}

We used the Hybrid Particle Lagrangian Integrated Trajectory (HYSPLIT) model to calculate 24-hour backward trajectories at $50 \mathrm{~m}$ above the ground from the sampling location for every hour during the campaign. Global Data Assimilation System's $0.5^{\circ} \times 0.5^{\circ}$ resolution product was used as the meteorological input. ${ }^{6}$ To find the potential geographical origins of VOCs measured in Berkeley, we performed Concentration-Weighted-Trajectory (CWT) analysis using the Igor Probased ZeFir Package. ${ }^{7}$ In the CWT analysis, the entire geographical region was divided into $0.05^{\circ}$ $\times 0.05^{\circ}$ cells. The concentrations measured at the receptor site were used as weighing factors for residence time of trajectories in each grid cell. This concentration-weighted residence time in each grid cell was then divided by the total residence time of all the trajectories in that cell to get the average weighted concentration of that cell. ${ }^{8}$ 


\section{Supporting measurements}

$\mathrm{PM}_{2.5}$ filter samples $(\mathrm{N}=74)$ with 3 to 4 -hour time resolution were collected concurrently at the same location. Details of the filter analysis are presented in Liang et al. ${ }^{9}$ In brief, we analyzed the chemical composition of these particle samples by two-dimensional gas chromatography coupled with high resolution mass spectrometry $(\mathrm{GC} \times \mathrm{GC}$ HRMS). Organic carbon $(\mathrm{OC})$ and elemental carbon (EC) of the filter samples were analyzed by a Sunset Laboratory Model 5 OC/EC Aerosol Analyzer using the NIOSH870 thermal protocol.

Hourly measurements of $\mathrm{NO}, \mathrm{NO}_{2}$ and $\mathrm{O}_{3}$ at air quality monitoring stations managed by the Bay Area Air Quality Management District (BAAQMD) were also used in this study (Figures S1 and S9). The measurement data can be accessed online at https://www.baaqmd.gov/about-airquality/current-air-quality/air-monitoring-data/. According to the monitoring network plan of BAAQMD, ${ }^{10} \mathrm{NO} / \mathrm{NO}_{2}$ were measured by Thermo Scientific TECO $42 \mathrm{i}$ chemiluminescence analyzers (federal reference method), with detection limit at $0.40 \mathrm{ppb}$ with 60 second averaging time. Ozone was measured by TECO 49i analyzers (UV photometric method, detection limit: 1 $\mathrm{ppb}$ ). Recent research reported positive artifacts of TECO 49i in the presence of biomass burning VOCs. ${ }^{11}$ Although at most sites the smoke plumes we encountered were less dense than those considered by Long et al., it is important to verify whether this could be the cause of enhanced $\mathrm{O}_{3}$ on plume days. Figure S10 shows the ozone and CO (a proxy of BB VOC during wildfire smoke events) level at the Napa site, which had the highest enhancement of CO due to wildfires. We did not observe abrupt increases of $\mathrm{O}_{3}$ mixing ratio simultaneously with the peaks of $\mathrm{CO}$. Also, as shown in Figure S9, the enhancement of ozone and $\mathrm{O}_{x}$ on plume days at sites very far away from the fire (Oakland East and San Jose) is comparable or even larger than that at the Napa site, which further suggests the enhancement of ozone on plume days cannot be explained by the artifacts of 
BB VOCs. Since the results by Long et al. is largely driven by data points in very dense smoke, we think more collocated measurements of chemiluminescence ozone analyzers with UV photometric analyzers in environments with lower levels of wildfire smoke are needed to better assess this interference.

\section{Estimation of the uncertainty of total $\mathrm{OH}$ reactivity of VOCs}

The uncertainty of the total $\mathrm{OH}$ reactivity of VOCs was estimated following the approach by Pfannerstill et al. (2019). ${ }^{12}$ Measurement uncertainties of compounds calibrated with standards are around $10 \%$. Compounds not calibrated with standards have quantification uncertainties of $30 \% .{ }^{13}$ For each mass, if one structure dominates the signal, we also assume a $10 \%$ uncertainty for the reaction rate constant with $\mathrm{OH}$. In cases where several very different chemical structures could be attributed to the measured mass, an $100 \%$ uncertainty of $\mathrm{kVOC}_{\mathrm{VOH}}$ was assumed to account for the possible large differences in $\mathrm{OH}$ reaction rate coefficients among isomers. ${ }^{12}$ Therefore, the uncertainty of the total calculated $\mathrm{OH}$ reactivity is dependent on whether compounds with high measurement and reaction rate uncertainties dominate the total reactivity. The $\mathrm{OH}$ reactivity of each compound, $\delta R_{O H}, i$, can be determined by error propagation:

$$
\frac{\delta R_{O H, i}}{R_{O H, i}}=\sqrt{\left(\frac{\delta k_{i}}{k_{i}}\right)^{2}+\left(\frac{\delta C_{i}}{C_{i}}\right)^{2}}
$$

where $\delta k_{i}$ is the uncertainty of the reaction rate coefficient of $\mathrm{VOC}_{\mathrm{i}}+\mathrm{OH}, \delta C_{i}$ is the uncertainty of the concentration of compound $i$. After getting $\delta R_{O H}, i / R_{O H, i}$, we calculated the uncertainty of total $\mathrm{OH}$ reactivity (by fraction) following:

$$
\frac{\delta \Sigma R_{O H, i}}{\Sigma R_{O H, i}}=\sqrt{\sum\left(C_{i} k_{i} \frac{\delta R_{O H, i}}{R_{O H, i}}\right)^{2}} / \sum C_{i} k_{i}
$$


Our calculation shows that the total $\mathrm{VOC}+\mathrm{OH}$ reactivity has an uncertainty of $20 \%, 22 \%$ and $24 \%\left(25^{\text {th }}\right.$ percentile, median, and $75^{\text {th }}$ percentile $)$. 


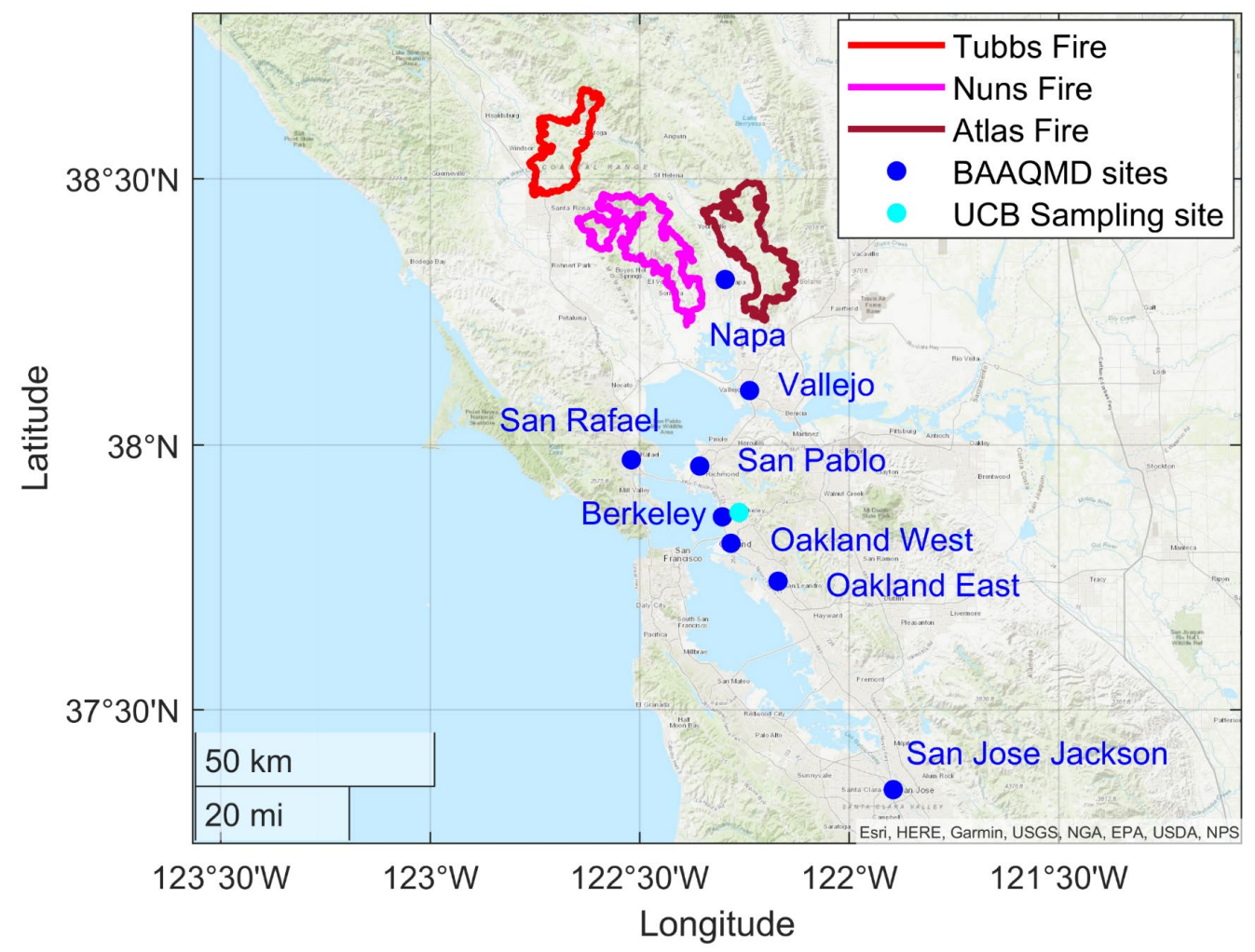

Figure S1. Perimeters of the major wildfires whose smoke affected Berkeley and selected Bay Area Air Quality Management District (BAAQMD) Air Quality Measurement Stations. 

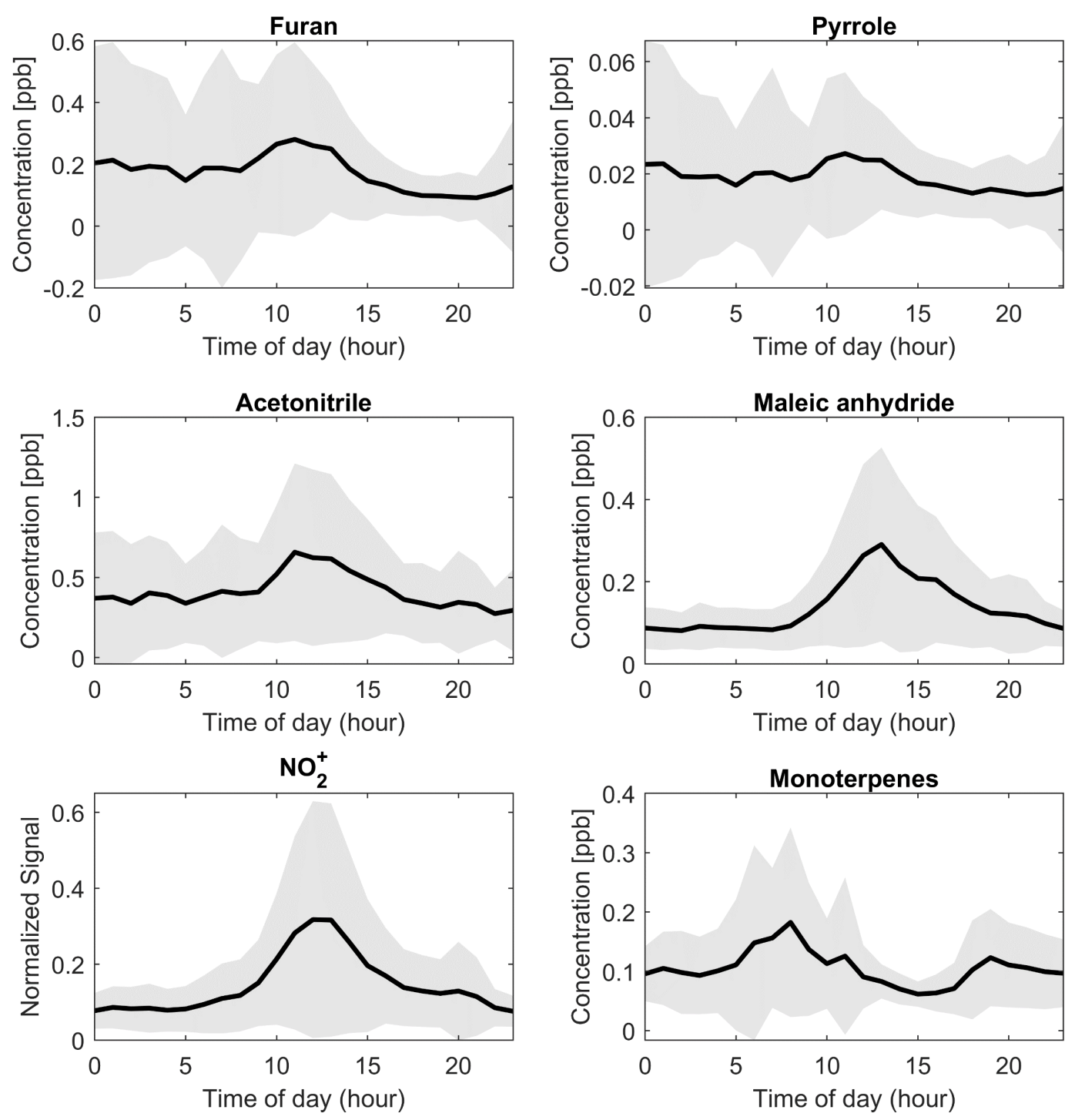

Figure S2. Diel plots of the concentrations of selected VOCs, and the normalized signal of $\mathrm{NO}_{2}{ }^{+}$ $(m / z=46)$. Solid line in black shows the mean and shaded area in gray represents one standard deviation. Time of day is local time (PDT). 

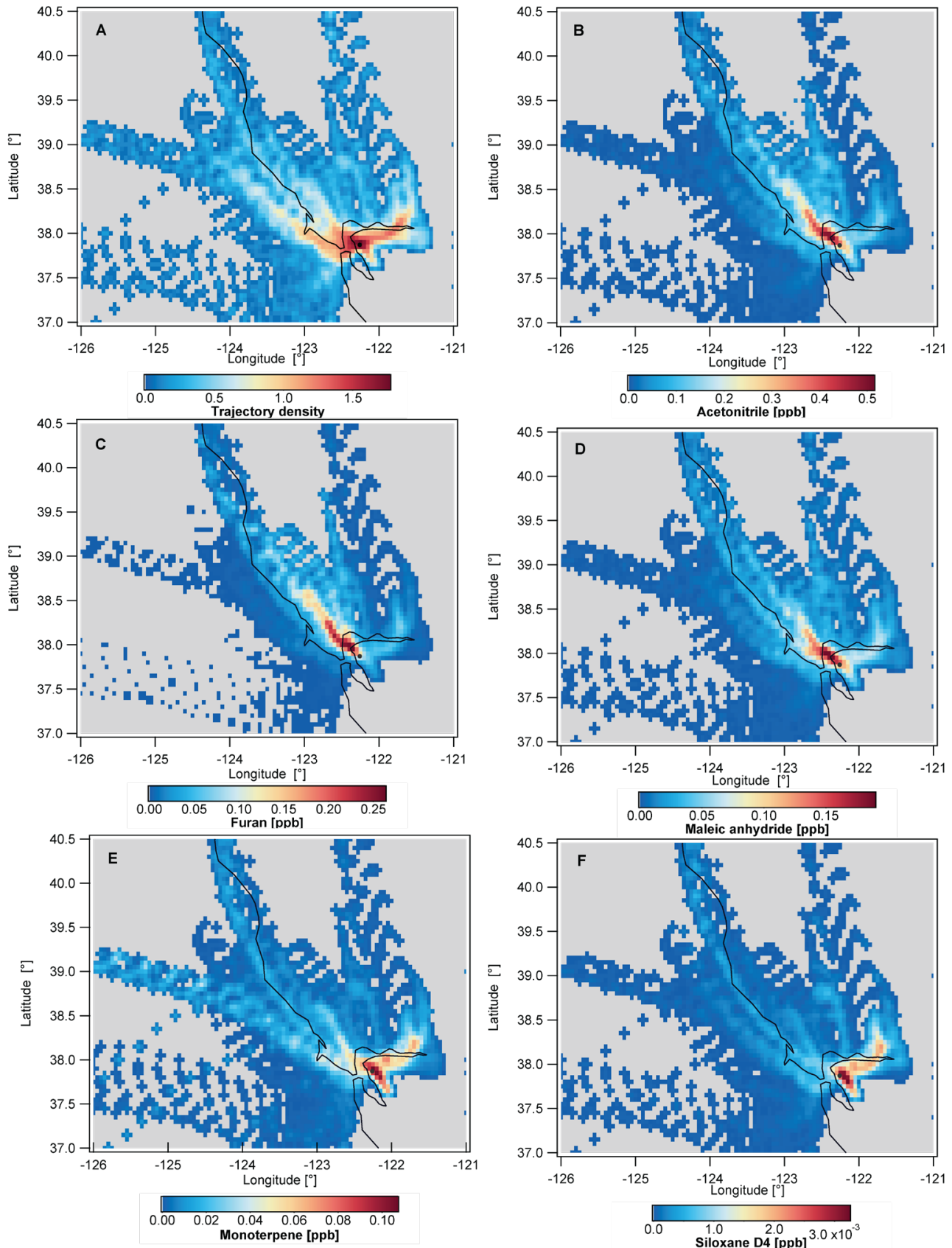

Figure S3. A. Concentration and B-F. Concentration Weighted Trajectories of individual VOCs: B. Acetonitrile; C. Furan; D. Maleic anhydride; E. Monoterpenes; and F. siloxane D4. The size of each grid cell is $0.05^{\circ} \times 0.05^{\circ}$. Black point shows the measurement site in Berkeley. 

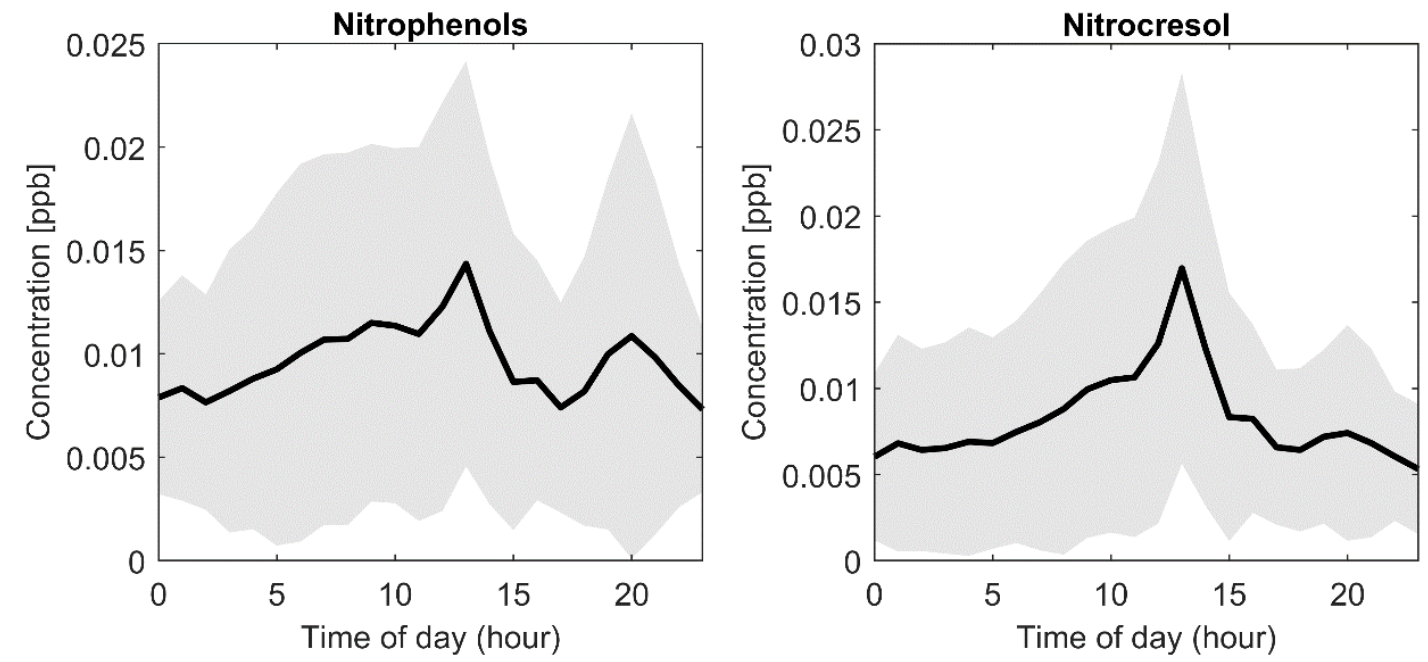

Figure S4. Diel plots of the concentrations of nitrophenols and nitrocresol. Solid line in black shows the mean and shaded area in gray represents one standard deviation. Time of day is local time (PDT). 


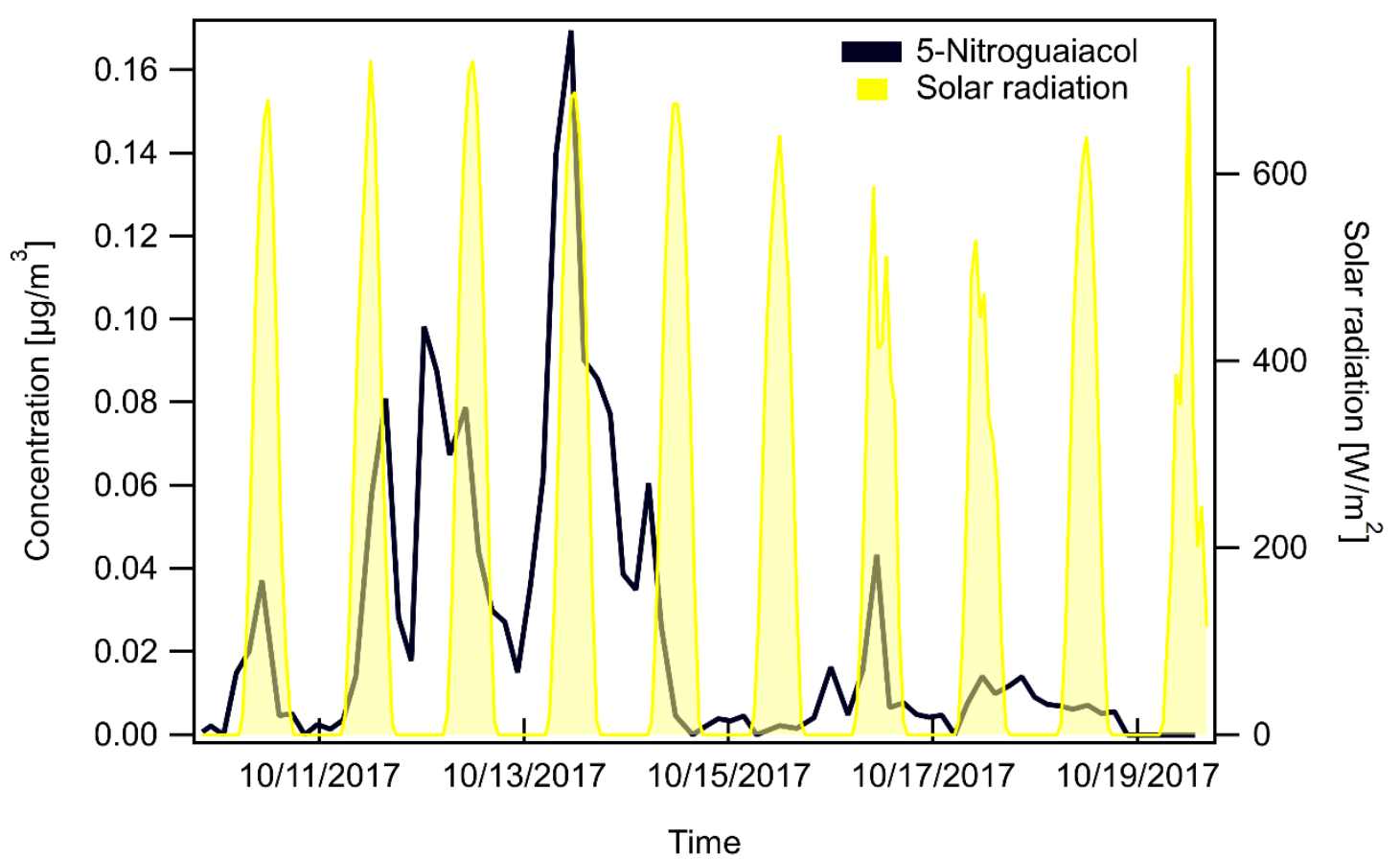

Figure S5 Particle phase 5-nitroguaiacol concentration measured by GC $\times$ GC-ToF-MS (left axis). Solar radiation measured at Bethel Island is shown in yellow (right axis). Time is in PDT. 

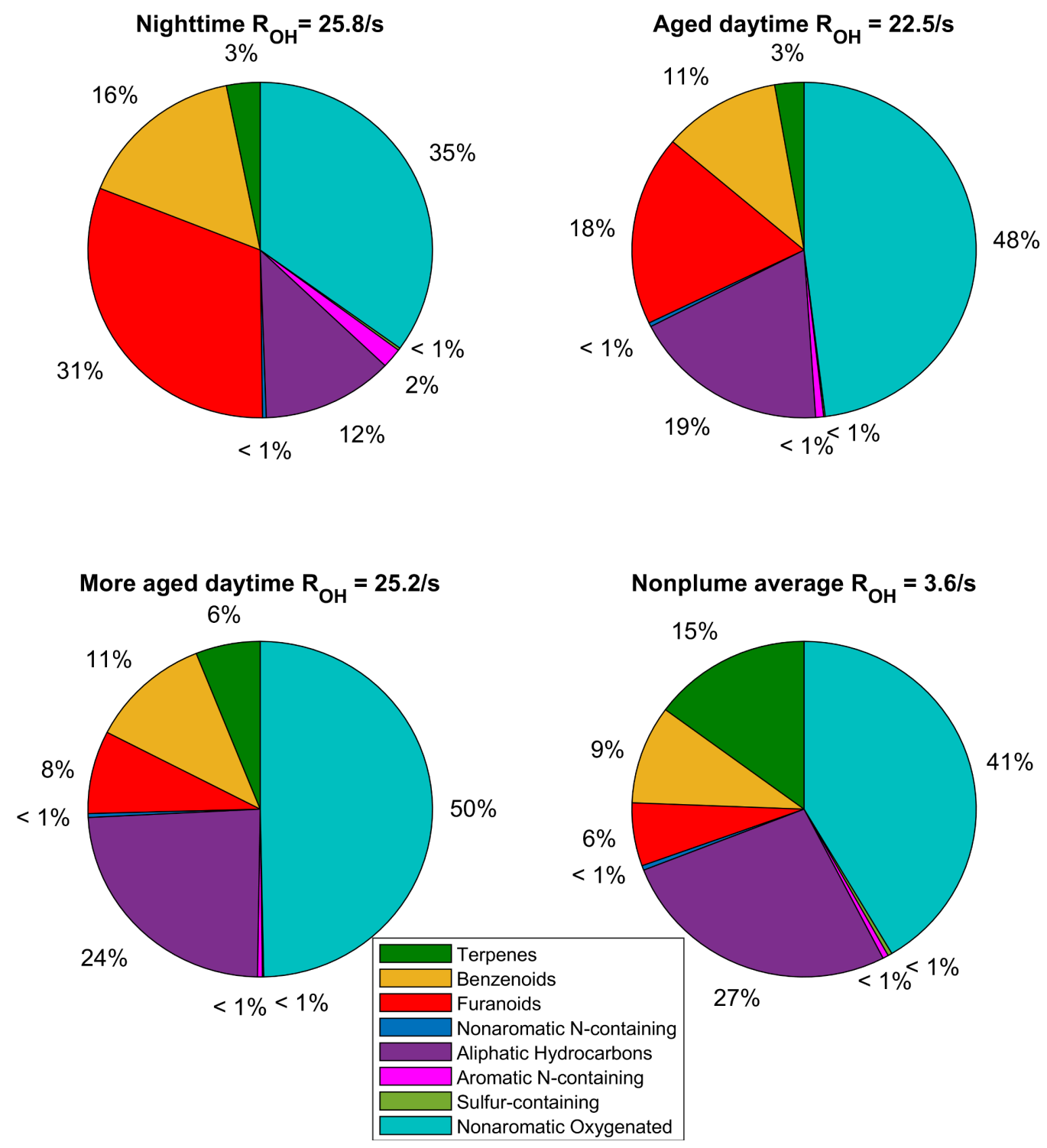

Figure S6. Organic OH reactivity contributed by different groups of VOCs in three plumes and the non-plume average. The relatively fresh nighttime plume and the aged daytime plume arrived at $0: 30$ and $13: 30$ on Oct 12, respectively. The more aged plume arrived at 11:00 on Oct 17. Nonplume average shows the average $\mathrm{OH}$ reactivities of VOCs on Oct 19 and Oct 20, when the influence of biomass burning plumes was very weak 


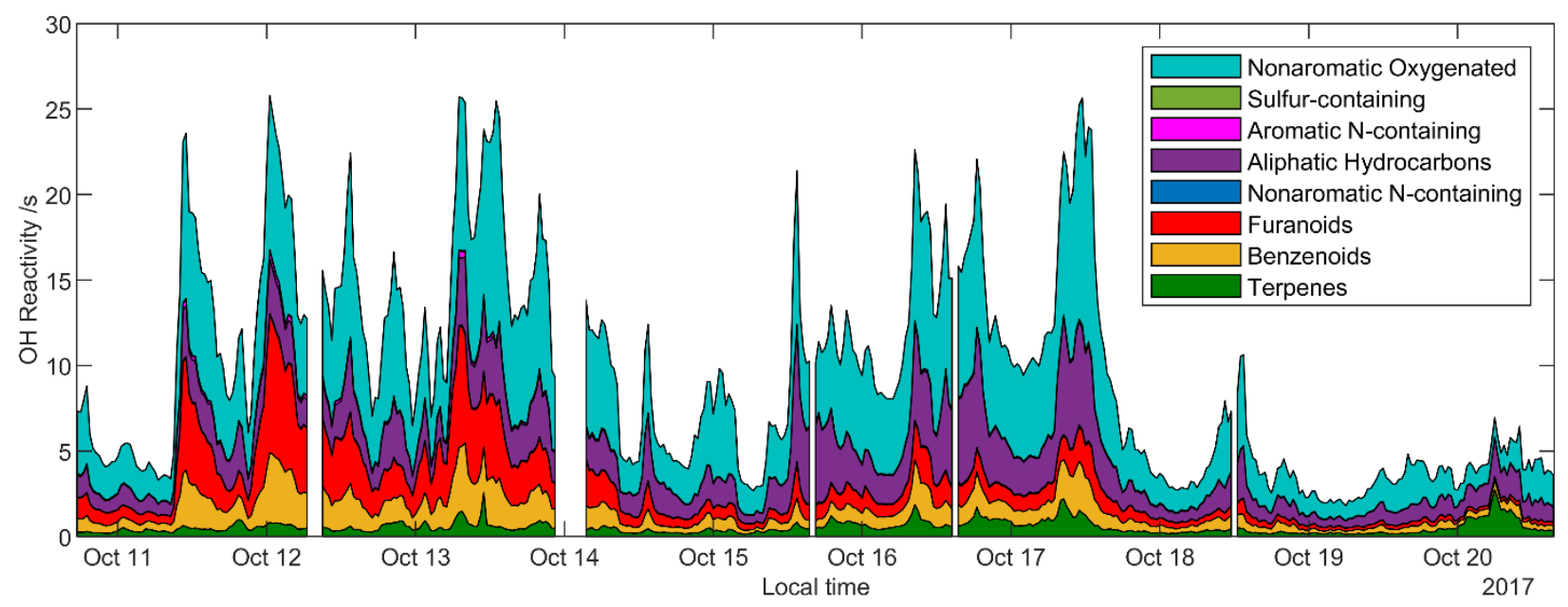

Figure S7. Time profile of organic OH reactivity contributed by different chemical families of VOCs. 


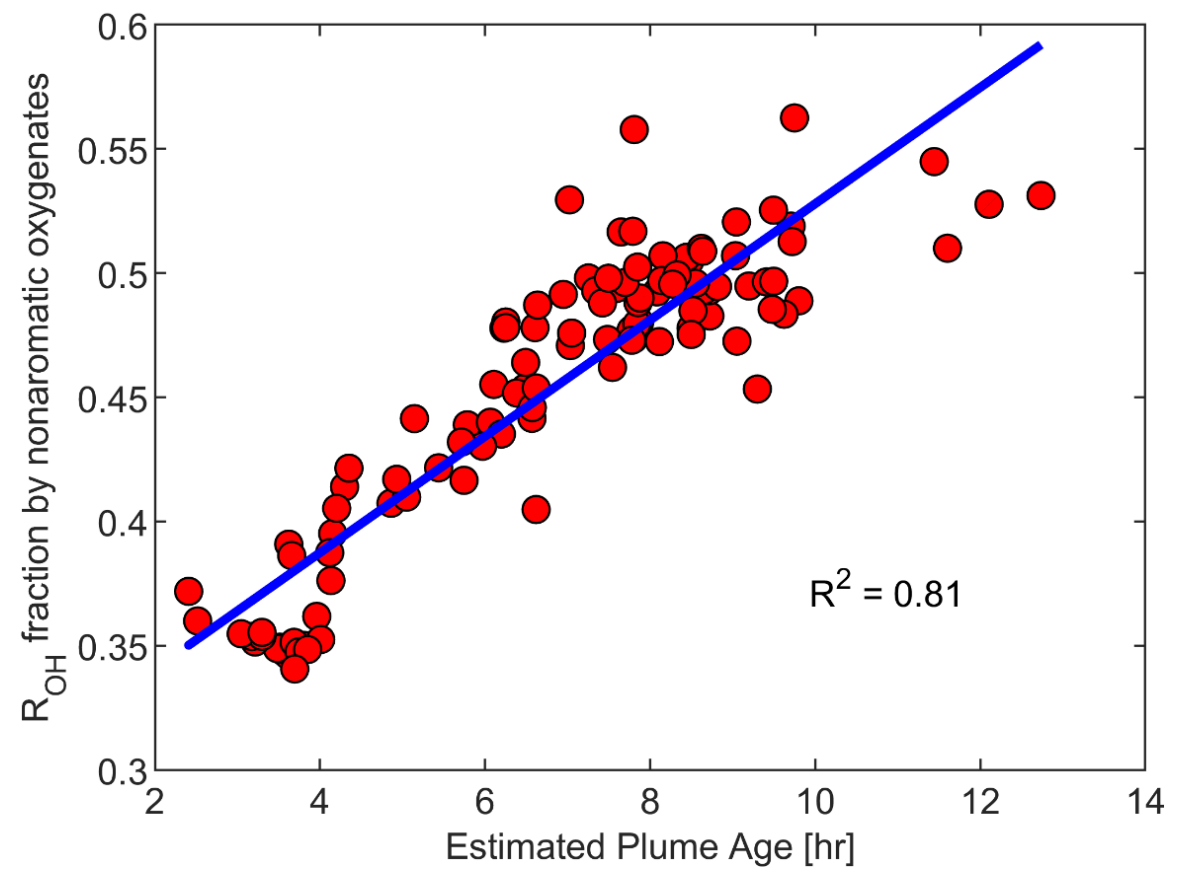

Figure S8. Fraction of total organic OH reactivity contributed by nonaromatic oxygenated VOCs vs. estimated chemical plume age. Plume age was calculated with the assumption that $[\mathrm{OH}]=$ $1.5 \times 10^{6}$ molec $\mathrm{cm}^{-3}$. 

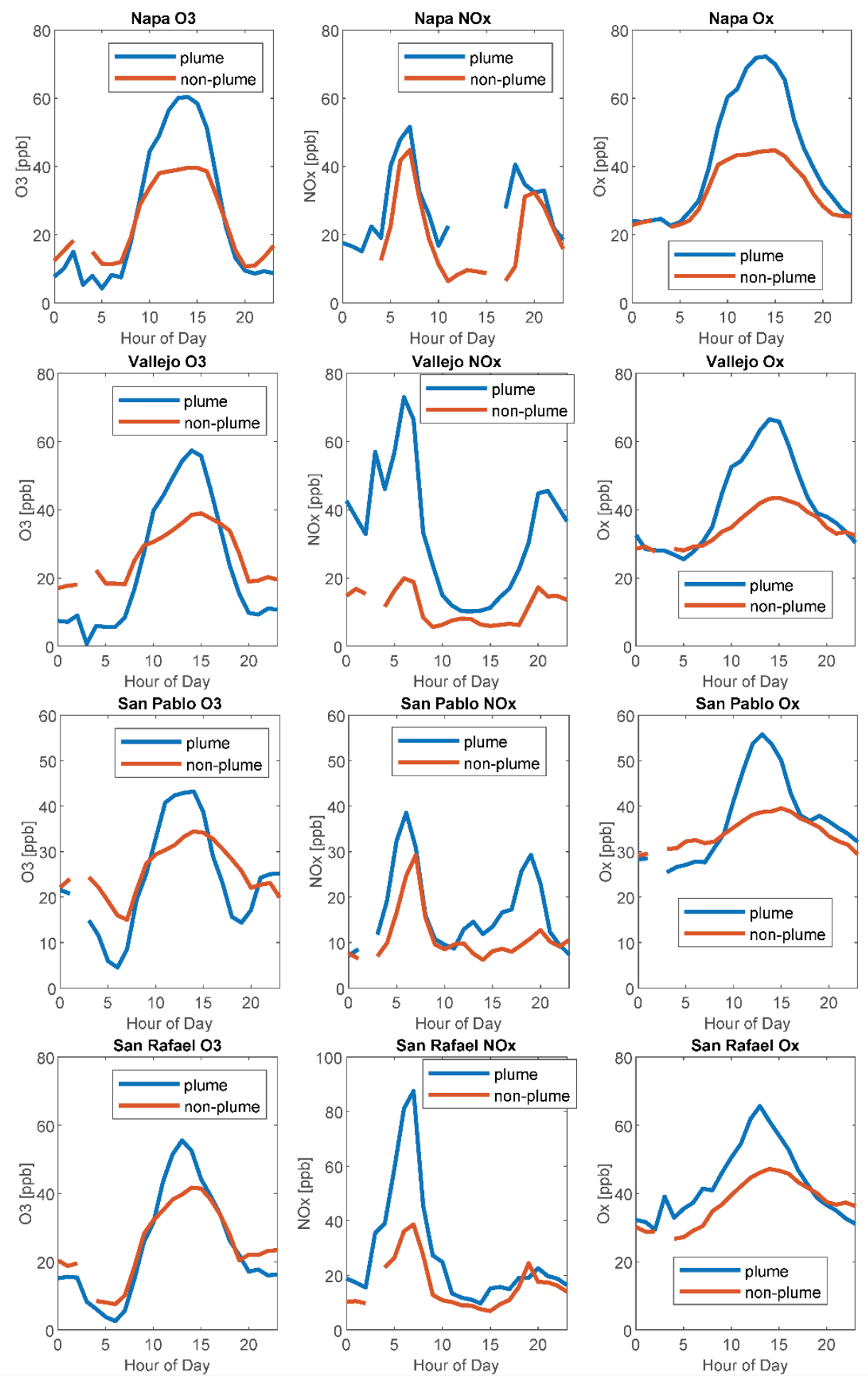

Figure S9a. Ozone, $\mathrm{NO}_{x}$ and $\mathrm{O}_{x}$ at selected BAAQMD measurement sites on plume days (Oct 1014, 16-17) and non-plume days (Oct 15, 18-20). Sites are ordered based on their distances from the fires (close to far). 

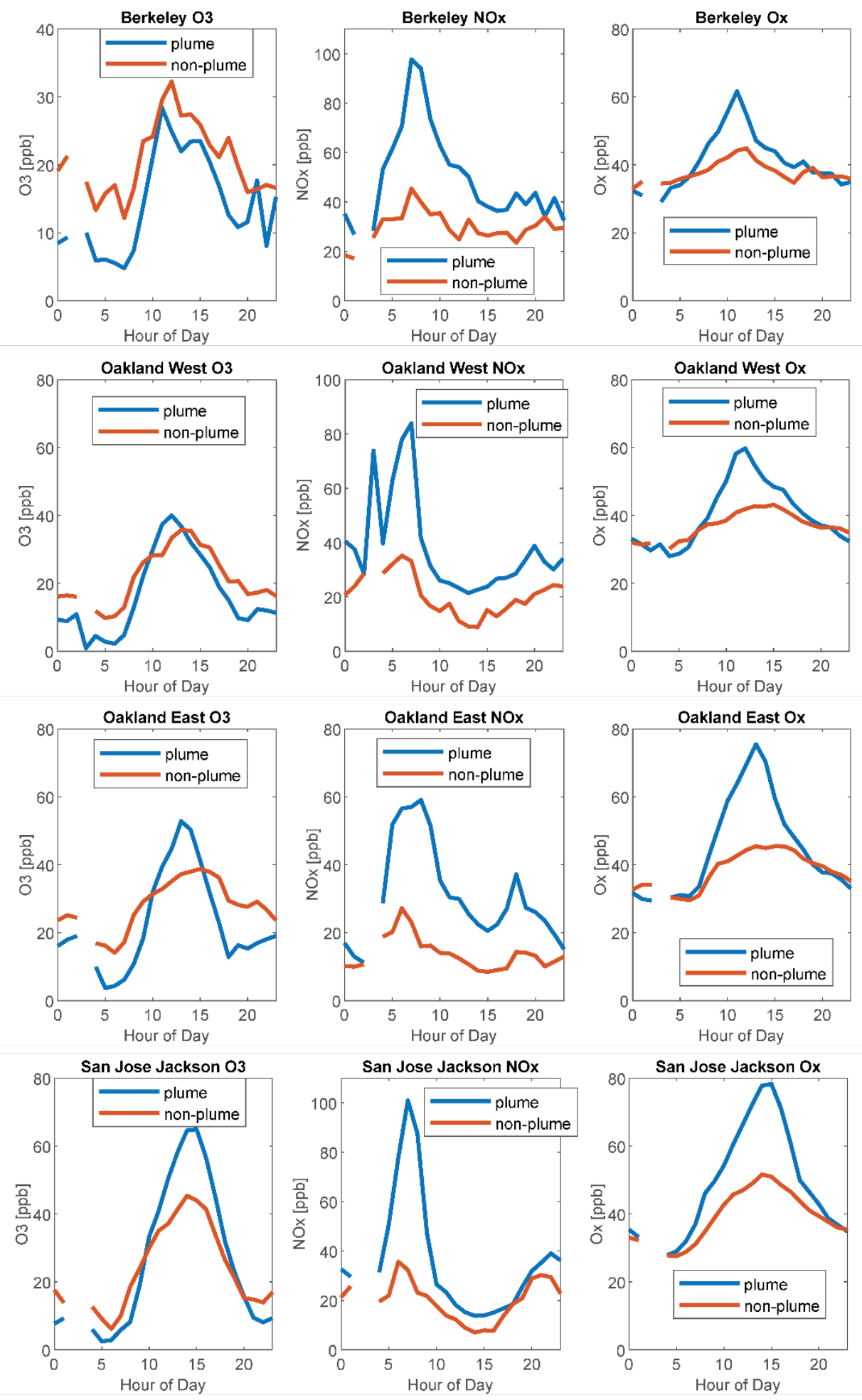

Figure S9b. Ozone, $\mathrm{NO}_{x}$ and $\mathrm{O}_{x}$ at selected BAAQMD measurement sites on plume days (Oct 1014, 16-17) and non-plume days (Oct 15, 18-20). Sites are ordered based on their distances from the fires (close to far). Continued from Fig. S9a. 


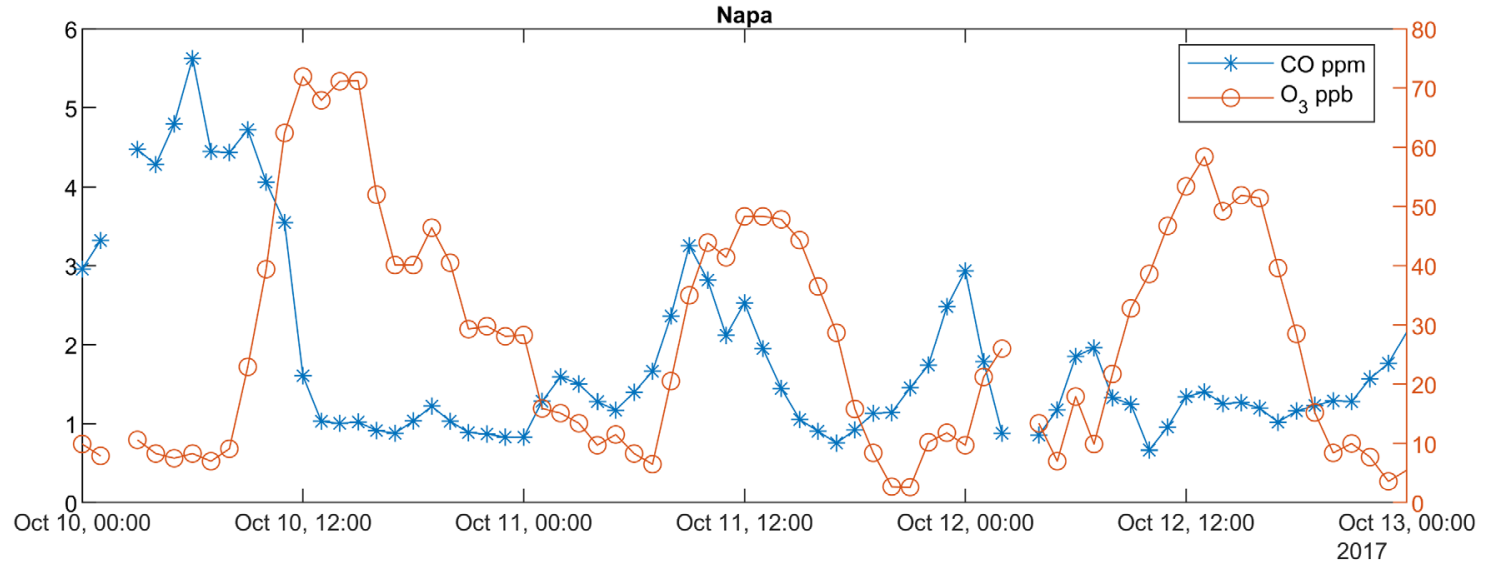

Figure S10. Mixing ratios of $\mathrm{CO}$ and ozone measured at the Napa measurement station during the strongest smoke plume events. 
Table S1 List of organic ions detected, compound assignment, and their coefficient of determination $\left(R^{2}\right)$ with furan and maleic anhydride.

\begin{tabular}{|c|c|c|c|c|}
\hline$m / z$ & Ion formula & $\begin{array}{c}\text { Tentative compound } \\
\text { assignment } \\
\end{array}$ & $\begin{array}{c}R^{2} \text { with } \\
\text { furan }\end{array}$ & $\begin{array}{c}\mathrm{R}^{2} \text { with maleic } \\
\text { anhydride }\end{array}$ \\
\hline 27.0227 & $\mathrm{C} 2 \mathrm{H} 3+$ & ethyne + alkyl fragment & 0.18 & 0.23 \\
\hline 31.0181 & $\mathrm{HCHOH}+$ & formaldehyde & 0.43 & 0.70 \\
\hline 33.0339 & $\mathrm{CH} 4 \mathrm{OH}+$ & methanol & 0.42 & 0.49 \\
\hline 41.0388 & $\mathrm{C} 3 \mathrm{H} 5+$ & 1,2-propadiene + alkyl fragment & 0.34 & 0.31 \\
\hline 42.034 & $\mathrm{C} 2 \mathrm{H} 3 \mathrm{NH}+$ & acetonitrile & 0.73 & 0.66 \\
\hline 43.018 & $\mathrm{C} 2 \mathrm{H} 3 \mathrm{O}+$ & acetic acid fragment & 0.50 & 0.75 \\
\hline 43.0537 & $\mathrm{C} 3 \mathrm{H} 7+$ & propene + propanol fragment & 0.15 & 0.18 \\
\hline 44.0124 & $\mathrm{CNHOH}+$ & isocyanic acid & 0.39 & 0.57 \\
\hline 45.0338 & $\mathrm{C} 2 \mathrm{H} 4 \mathrm{OH}+$ & acetaldehyde & 0.45 & 0.62 \\
\hline 45.9928 & $\mathrm{NO} 2+$ & $\begin{array}{c}\mathrm{NO}_{2}{ }^{+} \text {(fragment of organic } \\
\text { nitrates) }\end{array}$ & 0.22 & 0.86 \\
\hline 46.029 & $\mathrm{CH} 3 \mathrm{NOH}+$ & formamide & 0.22 & 0.64 \\
\hline 47.013 & $\mathrm{CH} 2 \mathrm{O} 2 \mathrm{H}+$ & formic acid & 0.08 & 0.50 \\
\hline 47.049 & $\mathrm{C} 2 \mathrm{H} 6 \mathrm{OH}+$ & ethanol & 0.00 & 0.10 \\
\hline 49.0124 & $\mathrm{CH} 4 \mathrm{SH}+$ & methanethiol & 0.66 & 0.22 \\
\hline 49.0272 & $\mathrm{CH} 4 \mathrm{O} 2 \mathrm{H}+$ & methane diol & 0.32 & 0.78 \\
\hline 51.9953 & $\mathrm{NH} 2 \mathrm{ClH}+$ & chloramine & 0.00 & 0.00 \\
\hline 53.038 & $\mathrm{C} 4 \mathrm{H} 4 \mathrm{H}+$ & 1-buten-3-yne & 0.80 & 0.38 \\
\hline 53.9924 & $\mathrm{NH}_{2}{ }^{37} \mathrm{ClH}+$ & chloramine $\left({ }^{37} \mathrm{Cl}\right)$ & 0.00 & 0.00 \\
\hline 54.0332 & $\mathrm{C} 3 \mathrm{H} 3 \mathrm{NH}+$ & acrylonitrile & 0.84 & 0.53 \\
\hline 56.0475 & $\mathrm{C} 3 \mathrm{H} 5 \mathrm{NH}+$ & propane nitrile & 0.73 & 0.55 \\
\hline 57.0332 & $\mathrm{C} 3 \mathrm{H} 4 \mathrm{OH}+$ & acrolein & 0.80 & 0.58 \\
\hline 57.0691 & C4H9+ & butenes + alkyl fragment & 0.16 & 0.23 \\
\hline 58.0293 & $\mathrm{C} 2 \mathrm{H} 3 \mathrm{NOH}+$ & $\begin{array}{l}\text { methyl isocyanate + hydroxy } \\
\text { acetonitrile }\end{array}$ & 0.55 & 0.62 \\
\hline 58.072 & $\mathrm{C} 3 \mathrm{H} 7 \mathrm{NH}^{+}$ & $\begin{array}{l}\text { 2-propen-1-amine }+2- \\
\text { propanimine }\end{array}$ & 0.17 & 0.25 \\
\hline 59.0484 & $\mathrm{C} 3 \mathrm{H} 6 \mathrm{OH}+$ & acetone + propanal & 0.13 & 0.36 \\
\hline 60.0467 & $\mathrm{C} 2 \mathrm{H} 5 \mathrm{NOH}+$ & acetamide & 0.26 & 0.59 \\
\hline 61.0276 & $\mathrm{C} 2 \mathrm{H} 4 \mathrm{O} 2 \mathrm{H}+$ & acetic acid & 0.52 & 0.72 \\
\hline 63.0255 & $\mathrm{C} 2 \mathrm{H} 6 \mathrm{SH}+$ & dimethyl sulfide & 0.01 & 0.00 \\
\hline 63.0418 & $\mathrm{C} 2 \mathrm{H} 6 \mathrm{O} 2 \mathrm{H}+$ & ethylene glycol & 0.12 & 0.31 \\
\hline 67.0534 & $\mathrm{C} 5 \mathrm{H} 6 \mathrm{H}+$ & 1,3-cyclopentadiene & 0.38 & 0.35 \\
\hline 68.0499 & $\mathrm{C} 4 \mathrm{H} 5 \mathrm{NH}+$ & pyrrole & 0.95 & 0.25 \\
\hline 65.0597 & $\mathrm{C} 4 \mathrm{H} 4 \mathrm{OH}+$ & furan & 1.00 & 0.28 \\
\hline 69.0697 & $\mathrm{C} 5 \mathrm{H} 8 \mathrm{H}+$ & isoprene +2 -methyl-3-buten-2-ol & 0.08 & 0.17 \\
\hline 70.0693 & $\mathrm{C} 4 \mathrm{H} 7 \mathrm{NH}+$ & butane nitrile + pyrroline & 0.40 & 0.50 \\
\hline
\end{tabular}




\begin{tabular}{|c|c|c|c|c|}
\hline 71.0483 & $\mathrm{C} 4 \mathrm{H} 6 \mathrm{OH}+$ & $\begin{array}{l}\text { methyl vinyl ketone }+ \\
\text { methacrolein }+ \text { crotonaldehyde }\end{array}$ & 0.36 & 0.30 \\
\hline 71.0849 & $\mathrm{C} 5 \mathrm{H} 10 \mathrm{H}+$ & C5H10 alkenes & 0.10 & 0.21 \\
\hline 72.0475 & $\mathrm{C} 3 \mathrm{H} 5 \mathrm{NOH}+$ & acrylamide & 0.32 & 0.34 \\
\hline 73.0277 & $\mathrm{C} 3 \mathrm{H} 4 \mathrm{O} 2 \mathrm{H}+$ & methylglyoxal + acrylic acid & 0.71 & 0.52 \\
\hline 73.0646 & $\mathrm{C} 4 \mathrm{H} 8 \mathrm{OH}+$ & methyl ethyl ketone + butanals & 0.45 & 0.60 \\
\hline 74.0242 & $\mathrm{C} 2 \mathrm{H} 3 \mathrm{NO} 2 \mathrm{H}+$ & nitroethene & 0.43 & 0.64 \\
\hline 75.0438 & $\mathrm{C} 3 \mathrm{H} 6 \mathrm{O} 2 \mathrm{H}+$ & $\begin{array}{l}\text { hydroxyacetone }+ \text { propanoic acid } \\
+ \text { methyl acetate }+ \text { ethyl formate }\end{array}$ & 0.59 & 0.70 \\
\hline 77.0231 & $\mathrm{C} 2 \mathrm{H} 4 \mathrm{O} 3 \mathrm{H}+$ & glycolic acid + PAN fragment & 0.22 & 0.85 \\
\hline 77.04 & $\mathrm{C} 3 \mathrm{H} 5 \mathrm{FOH}+$ & $\mathrm{C} 3 \mathrm{H} 5 \mathrm{FO}$ & 0.46 & 0.63 \\
\hline 79.0544 & C6H6H+ & benzene & 0.61 & 0.58 \\
\hline 80.051 & $\mathrm{C} 5 \mathrm{H} 5 \mathrm{NH}+$ & pyridine + pentadienenitriles & 0.77 & 0.63 \\
\hline 81.035 & $\mathrm{C} 5 \mathrm{H} 4 \mathrm{OH}+$ & 2,4-cyclopentadiene-1-one & 0.87 & 0.24 \\
\hline 81.0687 & $\mathrm{C} 6 \mathrm{H} 8 \mathrm{H}+$ & cyclohexadiene & 0.05 & 0.03 \\
\hline 82.0396 & $\mathrm{C} 3 \mathrm{H} 3 \mathrm{~N} 3 \mathrm{H}+$ & $\mathrm{C} 3 \mathrm{H} 3 \mathrm{~N} 3$ & 0.96 & 0.27 \\
\hline 83.0126 & $\mathrm{C} 4 \mathrm{H} 2 \mathrm{O} 2 \mathrm{H}+$ & $\mathrm{C} 4 \mathrm{H} 2 \mathrm{O} 2$ & 0.74 & 0.64 \\
\hline 83.0489 & $\mathrm{C} 5 \mathrm{H} 6 \mathrm{OH}+$ & methylfuran & 0.95 & 0.17 \\
\hline 83.0849 & $\mathrm{C} 6 \mathrm{H} 10 \mathrm{H}+$ & C6H10 & 0.08 & 0.19 \\
\hline 84.0462 & $\mathrm{C} 4 \mathrm{H} 5 \mathrm{NOH}+$ & $\mathrm{C} 4 \mathrm{H} 5 \mathrm{NO}$ & 0.95 & 0.37 \\
\hline 85.0278 & $\mathrm{C} 4 \mathrm{H} 4 \mathrm{O} 2 \mathrm{H}+$ & 2-(3H)furanone +2 -butenedial & 0.86 & 0.57 \\
\hline 85.0637 & $\mathrm{C} 5 \mathrm{H} 8 \mathrm{OH}+$ & $\begin{array}{l}\text { cyclopentanone + 3-methyl-3- } \\
\text { buten-2-one }\end{array}$ & 0.88 & 0.44 \\
\hline 85.0998 & $\mathrm{C} 6 \mathrm{H} 12 \mathrm{H}+$ & C6H12 & 0.19 & 0.23 \\
\hline 86.026 & $\mathrm{C} 3 \mathrm{H} 3 \mathrm{NO} 2 \mathrm{H}+$ & cyano acetic acid & 0.73 & 0.68 \\
\hline 86.0616 & $\mathrm{C} 4 \mathrm{H} 7 \mathrm{NOH}+$ & 2-pyrrolidinone & 0.69 & 0.60 \\
\hline 87.0432 & $\mathrm{C} 4 \mathrm{H} 6 \mathrm{O} 2 \mathrm{H}+$ & 2,3-butanedione & 0.82 & 0.55 \\
\hline 87.0797 & $\mathrm{C} 5 \mathrm{H} 10 \mathrm{OH}+$ & pentanones + pentanals + MBO & 0.65 & 0.60 \\
\hline 89.0261 & $\mathrm{C} 3 \mathrm{H} 4 \mathrm{O} 3 \mathrm{H}+$ & pyruvic acid & 0.36 & 0.79 \\
\hline 89.0595 & $\mathrm{C} 4 \mathrm{H} 8 \mathrm{O} 2 \mathrm{H}+$ & methyl propanoate +1 ,4-dioxane & 0.27 & 0.51 \\
\hline 93.0372 & $\mathrm{C} 3 \mathrm{H} 8 \mathrm{OSH}+$ & $\mathrm{C} 3 \mathrm{H} 8 \mathrm{OS}$ & 0.12 & 0.20 \\
\hline 93.0688 & $\mathrm{C} 7 \mathrm{H} 8 \mathrm{H}+$ & toluene & 0.18 & 0.26 \\
\hline 95.0482 & C6H6OH+ & phenol & 0.95 & 0.41 \\
\hline 95.0842 & $\mathrm{C} 7 \mathrm{H} 10 \mathrm{H}+$ & $\mathrm{C} 7 \mathrm{H} 10$ & 0.41 & 0.39 \\
\hline 96.9602 & $\mathrm{C}_{2} \mathrm{H}_{2}{ }^{35} \mathrm{Cl}_{2} \mathrm{H}+$ & dichloroethene & 0.55 & 0.15 \\
\hline 97.0277 & $\mathrm{C} 5 \mathrm{H} 4 \mathrm{O} 2 \mathrm{H}+$ & furfural & 0.98 & 0.30 \\
\hline 97.0639 & $\mathrm{C} 6 \mathrm{H} 8 \mathrm{OH}+$ & 2-ethylfuran $+2,5$-dimethylfuran & 0.98 & 0.27 \\
\hline 97.1014 & $\mathrm{C} 7 \mathrm{H} 12 \mathrm{H}+$ & methylcyclohexene & 0.15 & 0.29 \\
\hline 98.0256 & $\mathrm{C} 4 \mathrm{H} 3 \mathrm{NO} 2 \mathrm{H}+$ & maleimide & 0.92 & 0.48 \\
\hline 99.0085 & $\mathrm{C} 4 \mathrm{H} 2 \mathrm{O} 3 \mathrm{H}+$ & maleic anhydride & 0.28 & 1.00 \\
\hline 99.043 & $\mathrm{C} 5 \mathrm{H} 6 \mathrm{O} 2 \mathrm{H}+$ & furfuryl alcohol & 0.85 & 0.55 \\
\hline 99.0789 & $\mathrm{C} 6 \mathrm{H} 10 \mathrm{OH}+$ & hexenals + cyclohexanone & 0.50 & 0.46 \\
\hline 100.04 & $\mathrm{C} 4 \mathrm{H} 5 \mathrm{NO} 2 \mathrm{H}+$ & succinimide & 0.52 & 0.64 \\
\hline 100.075 & $\mathrm{C} 5 \mathrm{H} 9 \mathrm{NOH}+$ & $\begin{array}{c}\text { 1-methyl-2-pyrrolidinone }+2- \\
\text { piperidinone }\end{array}$ & 0.05 & 0.12 \\
\hline
\end{tabular}




\begin{tabular}{|c|c|c|c|c|}
\hline 101.024 & $\mathrm{C} 4 \mathrm{H} 4 \mathrm{O} 3 \mathrm{H}^{+}$ & succinic anhydride & 0.32 & 0.71 \\
\hline 101.059 & $\mathrm{C} 5 \mathrm{H} 8 \mathrm{O} 2 \mathrm{H}+$ & methyl methacrylate & 0.45 & 0.49 \\
\hline 101.094 & $\mathrm{C} 6 \mathrm{H} 12 \mathrm{OH}+$ & hexanals and hexanones & 0.28 & 0.42 \\
\hline 102.092 & $\mathrm{C} 5 \mathrm{H} 11 \mathrm{NOH}+$ & C5H11NO (e.g., pentanamide) & 0.38 & 0.58 \\
\hline 103.039 & $\mathrm{C} 4 \mathrm{H} 6 \mathrm{O} 3 \mathrm{H}^{+}$ & acetic anhydride & 0.70 & 0.74 \\
\hline 103.074 & $\mathrm{C} 5 \mathrm{H} 10 \mathrm{O} 2 \mathrm{H}+$ & pentanoic acid & 0.46 & 0.72 \\
\hline 104.047 & $\mathrm{C} 7 \mathrm{H} 5 \mathrm{NH}+$ & benzonitrile & 0.79 & 0.66 \\
\hline 105.036 & $\mathrm{C} 4 \mathrm{H} 8 \mathrm{OSH}+$ & methional & 0.56 & 0.78 \\
\hline 105.069 & $\mathrm{C} 8 \mathrm{H} 8 \mathrm{H}+$ & styrene & 0.49 & 0.24 \\
\hline 107.05 & $\mathrm{C} 7 \mathrm{H} 6 \mathrm{OH}+$ & benzaldehyde & 0.37 & 0.41 \\
\hline 107.086 & $\mathrm{C} 8 \mathrm{H} 10 \mathrm{H}+$ & $\begin{array}{c}\text { C8 aromatics, mainly xylene and } \\
\text { ethylbenzene }\end{array}$ & 0.05 & 0.12 \\
\hline 109.028 & $\mathrm{C} 6 \mathrm{H} 4 \mathrm{O} 2 \mathrm{H}+$ & p-benzoquinone & 0.79 & 0.69 \\
\hline 109.065 & $\mathrm{C} 7 \mathrm{H} 8 \mathrm{OH}+$ & cresol & 0.95 & 0.29 \\
\hline 109.1 & $\mathrm{C} 8 \mathrm{H} 12 \mathrm{H}+$ & $\mathrm{C} 8 \mathrm{H} 12$ & 0.37 & 0.42 \\
\hline 111.044 & $\mathrm{C} 6 \mathrm{H} 6 \mathrm{O} 2 \mathrm{H}+$ & benzenediols + methylfurfurals & 0.98 & 0.26 \\
\hline 111.08 & $\mathrm{C} 7 \mathrm{H} 10 \mathrm{OH}+$ & trimethylfuran + norcamphor & 0.86 & 0.28 \\
\hline 111.116 & $\mathrm{C} 8 \mathrm{H} 14 \mathrm{H}+$ & $\mathrm{C} 8 \mathrm{H} 14$ & 0.01 & 0.08 \\
\hline 112.041 & $\mathrm{C} 5 \mathrm{H} 5 \mathrm{NO} 2 \mathrm{H}+$ & $\begin{array}{c}\text { dihydroxy pyridine + methyl } \\
\text { maleimide }\end{array}$ & 0.87 & 0.59 \\
\hline 113.024 & $\mathrm{C} 5 \mathrm{H} 4 \mathrm{O} 3 \mathrm{H}+$ & $\begin{array}{c}\text { 5-hydroxy 2-furfural+2-furoic } \\
\text { acid+citraconic anhydride }\end{array}$ & 0.53 & 0.91 \\
\hline 113.059 & $\mathrm{C} 6 \mathrm{H} 8 \mathrm{O} 2 \mathrm{H}+$ & $\begin{array}{c}\text { 2-hydroxy-3-methyl-2- } \\
\text { cyclopenten-1-one }\end{array}$ & 0.85 & 0.56 \\
\hline 113.094 & $\mathrm{C} 7 \mathrm{H} 12 \mathrm{OH}+$ & ethylcyclopentanone & 0.39 & 0.51 \\
\hline 114.056 & $\mathrm{C} 5 \mathrm{H} 7 \mathrm{NO} 2 \mathrm{H}+$ & $\mathrm{C} 5 \mathrm{H} 7 \mathrm{NO} 2$ & 0.59 & 0.71 \\
\hline 114.092 & $\mathrm{C} 6 \mathrm{H} 11 \mathrm{NOH}+$ & caprolactam & 0.27 & 0.45 \\
\hline 115.04 & $\mathrm{C} 5 \mathrm{H} 6 \mathrm{O} 3 \mathrm{H}+$ & 5-hydroxymethyl-2-furanone & 0.57 & 0.80 \\
\hline 115.074 & $\mathrm{C} 6 \mathrm{H} 10 \mathrm{O} 2 \mathrm{H}+$ & C6H10O2 (e.g., hexenoic acid) & 0.31 & 0.59 \\
\hline 115.109 & $\mathrm{C} 7 \mathrm{H} 14 \mathrm{OH}+$ & heptanal & 0.13 & 0.43 \\
\hline 117.055 & $\mathrm{C} 5 \mathrm{H} 8 \mathrm{O} 3 \mathrm{H}+$ & $\begin{array}{c}\text { 5-hydroxymethyl tetrahydro 2- } \\
\text { furanone+5-hydroxy tetrahydro } \\
\text { 2-furfural }\end{array}$ & 0.72 & 0.74 \\
\hline 117.089 & $\mathrm{C} 6 \mathrm{H} 12 \mathrm{O} 2 \mathrm{H}+$ & C6 acid + esters & 0.14 & 0.42 \\
\hline 118.062 & $\mathrm{C} 8 \mathrm{H} 7 \mathrm{NH}+$ & benzonitrile, 3-methyl- & 0.73 & 0.66 \\
\hline 118.903 & $\mathrm{C}^{35} \mathrm{Cl}_{3} \mathrm{H}+$ & CCl4 fragment & 0.01 & 0.00 \\
\hline 119.049 & $\mathrm{C} 8 \mathrm{H} 6 \mathrm{OH}+$ & benzofuran & 0.94 & 0.45 \\
\hline 119.084 & $\mathrm{C} 9 \mathrm{H} 10 \mathrm{H}+$ & methyl styrenes & 0.39 & 0.26 \\
\hline 120.9 & $\mathrm{C}^{35} \mathrm{Cl}_{2}^{37} \mathrm{ClH}+$ & CCl4 fragment & 0.04 & 0.03 \\
\hline 121.1 & $\mathrm{C} 9 \mathrm{H} 12 \mathrm{H}+$ & C9 aromatics & 0.03 & 0.09 \\
\hline 122.024 & $\mathrm{C} 2 \mathrm{H} 3 \mathrm{NO} 5 \mathrm{H}+$ & $\begin{array}{c}\text { peroxyacetyl nitrate (PAN) } \\
\text { parent ion }\end{array}$ & 0.30 & 0.76 \\
\hline 122.063 & $\mathrm{C} 7 \mathrm{H} 7 \mathrm{NOH}+$ & $\mathrm{C} 7 \mathrm{H} 7 \mathrm{NO}$ & 0.66 & 0.63 \\
\hline 123.044 & $\mathrm{C} 7 \mathrm{H} 6 \mathrm{O} 2 \mathrm{H}+$ & $\begin{array}{l}\text { 2-hydroxybenzaldehyde+ benzoic } \\
\text { acid }\end{array}$ & 0.65 & 0.74 \\
\hline 123.079 & $\mathrm{C} 8 \mathrm{H} 10 \mathrm{OH}+$ & methyl anisole & 0.96 & 0.28 \\
\hline
\end{tabular}




\begin{tabular}{|c|c|c|c|c|}
\hline 123.116 & $\mathrm{C} 9 \mathrm{H} 14 \mathrm{H}+$ & С9H14 & 0.27 & 0.29 \\
\hline 125.025 & $\mathrm{C} 6 \mathrm{H} 4 \mathrm{O} 3 \mathrm{H}+$ & $\begin{array}{l}\text { hydroxy benzoquinone }+ \\
\text { diformylfuran }\end{array}$ & 0.87 & 0.52 \\
\hline 125.06 & $\mathrm{C} 7 \mathrm{H} 8 \mathrm{O} 2 \mathrm{H}+$ & guaiacol & 0.97 & 0.22 \\
\hline 125.096 & $\mathrm{C} 8 \mathrm{H} 12 \mathrm{OH}+$ & furan, 4-methyl-2-propyl- & 0.88 & 0.32 \\
\hline 125.131 & $\mathrm{C} 9 \mathrm{H} 16 \mathrm{H}+$ & 3,3,5-trimethylcyclohexene & 0.03 & 0.17 \\
\hline 127.04 & $\mathrm{C} 6 \mathrm{H} 6 \mathrm{O} 3 \mathrm{H}+$ & 5-(hydroxymethyl)-2-furfural & 0.77 & 0.70 \\
\hline 127.075 & $\mathrm{C} 7 \mathrm{H} 10 \mathrm{O} 2 \mathrm{H}+$ & $\mathrm{C} 7 \mathrm{H} 10 \mathrm{O} 2$ & 0.63 & 0.61 \\
\hline 127.111 & $\mathrm{C} 8 \mathrm{H} 14 \mathrm{OH}+$ & trans-2-octenal & 0.03 & 0.18 \\
\hline 129.059 & $\mathrm{C} 6 \mathrm{H} 8 \mathrm{O} 3 \mathrm{H}+$ & 2,5-di(hydroxymethyl)furan & 0.68 & 0.65 \\
\hline 129.088 & $\mathrm{C} 7 \mathrm{H} 12 \mathrm{O} 2 \mathrm{H}+$ & C7H12O2 (e.g., heptenoic acid) & 0.44 & 0.55 \\
\hline 131.037 & $\mathrm{C} 5 \mathrm{H} 6 \mathrm{O} 4 \mathrm{H}+$ & 2-pentenedioic acid & 0.65 & 0.77 \\
\hline 131.071 & $\mathrm{C} 6 \mathrm{H} 10 \mathrm{O} 3 \mathrm{H}+$ & $\begin{array}{l}\text { C6H10O3 (e.g., propionic } \\
\text { anhydride and hydroxyethyl } \\
\text { methacrylate) }\end{array}$ & 0.71 & 0.69 \\
\hline 131.104 & $\mathrm{C} 7 \mathrm{H} 14 \mathrm{O} 2 \mathrm{H}+$ & C7 acids+esters & 0.16 & 0.44 \\
\hline 132.078 & C9H9NH+ & methyl benzeneacetonitrile & 0.78 & 0.66 \\
\hline 133.064 & $\mathrm{C} 9 \mathrm{H} 8 \mathrm{OH}+$ & $\begin{array}{l}\text { methyl (iso)benzofuran + } \\
\text { cinnamaldehyde }\end{array}$ & 0.94 & 0.41 \\
\hline 133.1 & $\mathrm{C} 10 \mathrm{H} 12 \mathrm{H}+$ & $\begin{array}{c}\text { methyl propenyl benzene }+ \text { ethyl } \\
\text { styrene }\end{array}$ & 0.37 & 0.25 \\
\hline 135.048 & $\mathrm{C} 8 \mathrm{H} 6 \mathrm{O} 2 \mathrm{H}+$ & phthalaldehyde + phthalide & 0.55 & 0.70 \\
\hline 135.081 & $\mathrm{C} 9 \mathrm{H} 10 \mathrm{OH}+$ & 3-methylacetophenone & 0.42 & 0.53 \\
\hline 135.116 & $\mathrm{C} 10 \mathrm{H} 14 \mathrm{H}+$ & $\mathrm{C} 10$ aromatics & 0.03 & 0.15 \\
\hline 136.026 & $\mathrm{C} 2 \mathrm{H} 5 \mathrm{C}(\mathrm{O}) \mathrm{O} 2 \mathrm{NO} 2 \mathrm{H}+$ & peroxypropionyl nitrate (PPN) & 0.11 & 0.36 \\
\hline 136.038 & $\mathrm{C} 7 \mathrm{H} 5 \mathrm{NO} 2 \mathrm{H}+$ & 2-furoylacetonitrile & 0.40 & 0.69 \\
\hline 136.078 & $\mathrm{C} 8 \mathrm{H} 9 \mathrm{NOH}+$ & 2-aminoacetophenone & 0.61 & 0.66 \\
\hline 137.061 & $\mathrm{C} 8 \mathrm{H} 8 \mathrm{O} 2 \mathrm{H}+$ & methyl benzoic acid & 0.78 & 0.59 \\
\hline 137.098 & $\mathrm{C} 9 \mathrm{H} 12 \mathrm{OH}+$ & $\begin{array}{c}\text { 2,3-dimethylanisole }+4 \text {-ethyl } \\
\text { anisole }\end{array}$ & 0.45 & 0.15 \\
\hline 137.132 & $\mathrm{C} 10 \mathrm{H} 16 \mathrm{H}+$ & monoterpenes & 0.02 & 0.00 \\
\hline 139.041 & $\mathrm{C} 7 \mathrm{H} 6 \mathrm{O} 3 \mathrm{H}+$ & hydroxybenzoic acids & 0.81 & 0.59 \\
\hline 139.075 & $\mathrm{C} 8 \mathrm{H} 10 \mathrm{O} 2 \mathrm{H}+$ & creosol & 0.94 & 0.25 \\
\hline 139.111 & $\mathrm{C} 9 \mathrm{H} 14 \mathrm{OH}+$ & nopinone & 0.48 & 0.41 \\
\hline 140.036 & $\mathrm{C} 6 \mathrm{H} 5 \mathrm{NO} 3 \mathrm{H}+$ & nitrophenols & 0.41 & 0.57 \\
\hline 141.056 & $\mathrm{C} 7 \mathrm{H} 8 \mathrm{O} 3 \mathrm{H}+$ & methoxycatechol & 0.67 & 0.74 \\
\hline 141.091 & $\mathrm{C} 8 \mathrm{H} 12 \mathrm{O} 2 \mathrm{H}+$ & C8H12O2 (e.g., octdienoic acid) & 0.41 & 0.59 \\
\hline 141.126 & $\mathrm{C} 9 \mathrm{H} 16 \mathrm{OH}+$ & C9H16O (e.g., nonenal) & 0.04 & 0.26 \\
\hline 143.038 & $\mathrm{C} 6 \mathrm{H} 6 \mathrm{O} 4 \mathrm{H}+$ & C6H6O4 (e.g., benzenetetrols) & 0.60 & 0.77 \\
\hline 143.106 & $\mathrm{C} 8 \mathrm{H} 14 \mathrm{O} 2 \mathrm{H}+$ & C8H14O2 (e.g., octenoid acid) & 0.11 & 0.31 \\
\hline 143.141 & $\mathrm{C} 9 \mathrm{H} 18 \mathrm{OH}+$ & nonanal +2 -nonanone & 0.01 & 0.14 \\
\hline 145.051 & $\mathrm{C} 6 \mathrm{H} 8 \mathrm{O} 4 \mathrm{H}+$ & $\begin{array}{c}\text { levoglucosan dehydration } \\
\text { product }\end{array}$ & 0.66 & 0.72 \\
\hline 145.121 & $\mathrm{C} 8 \mathrm{H} 16 \mathrm{O} 2 \mathrm{H}+$ & $\mathrm{C} 8$ acid + esters & 0.03 & 0.21 \\
\hline 146.977 & $\mathrm{C} 6 \mathrm{H} 4 \mathrm{Cl} 2 \mathrm{H}+$ & dichlorobenzene & 0.04 & 0.05 \\
\hline 147.066 & $\mathrm{C} 6 \mathrm{H} 10 \mathrm{O} 4 \mathrm{H}+$ & adipic acid & 0.68 & 0.47 \\
\hline
\end{tabular}




\begin{tabular}{|c|c|c|c|c|}
\hline 147.078 & $\mathrm{C} 10 \mathrm{H} 10 \mathrm{OH}+$ & dimethylbenzofuran & 0.91 & 0.37 \\
\hline 147.114 & $\mathrm{C} 11 \mathrm{H} 14 \mathrm{H}+$ & $\mathrm{C} 11 \mathrm{H} 14$ & 0.48 & 0.30 \\
\hline 149.03 & $\mathrm{C} 8 \mathrm{H} 4 \mathrm{O} 3 \mathrm{H}+$ & phthalic anhydride & 0.07 & 0.23 \\
\hline 149.13 & $\mathrm{C} 11 \mathrm{H} 16 \mathrm{H}+$ & $\mathrm{C} 11 \mathrm{H} 16$ & 0.17 & 0.27 \\
\hline 151.077 & $\mathrm{C} 9 \mathrm{H} 10 \mathrm{O} 2 \mathrm{H}+$ & vinyl guaiacol & 0.82 & 0.48 \\
\hline 152.075 & $\mathrm{C} 8 \mathrm{H} 9 \mathrm{NO} 2 \mathrm{H}+$ & methyl anthranilate & 0.85 & 0.46 \\
\hline 153.057 & $\mathrm{C} 8 \mathrm{H} 8 \mathrm{O} 3 \mathrm{H}+$ & methyl salicylate + vanillin & 0.66 & 0.53 \\
\hline 154.054 & $\mathrm{C} 7 \mathrm{H} 7 \mathrm{NO} 3 \mathrm{H}+$ & nitrocresol & 0.58 & 0.71 \\
\hline 155.14 & $\mathrm{C} 10 \mathrm{H} 18 \mathrm{OH}+$ & cineole & 0.12 & 0.34 \\
\hline 157.155 & $\mathrm{C} 10 \mathrm{H} 20 \mathrm{OH}+$ & decanal & 0.11 & 0.42 \\
\hline 159.068 & $\mathrm{C} 7 \mathrm{H} 10 \mathrm{O} 4 \mathrm{H}+$ & $\mathrm{C} 7 \mathrm{H} 10 \mathrm{O} 4$ & 0.27 & 0.44 \\
\hline 159.136 & $\mathrm{C} 9 \mathrm{H} 18 \mathrm{O} 2 \mathrm{H}+$ & methyl octanoate & 0.00 & 0.12 \\
\hline 160.998 & $\mathrm{C} 7 \mathrm{H} 3{ }^{35} \mathrm{ClF} 2 \mathrm{H}+$ & $\begin{array}{l}\text { parachlorobenzotrifluoride } \\
\text { (PCBTF) fragment }\end{array}$ & 0.00 & 0.05 \\
\hline 161.057 & $\mathrm{C} 10 \mathrm{H} 8 \mathrm{O} 2 \mathrm{H}+$ & 2-formylcinnamaldehyde & 0.36 & 0.58 \\
\hline 161.093 & $\mathrm{C} 11 \mathrm{H} 12 \mathrm{OH}+$ & $\mathrm{C} 11 \mathrm{H} 12 \mathrm{O}$ & 0.81 & 0.52 \\
\hline 161.13 & $\mathrm{C} 12 \mathrm{H} 16 \mathrm{H}+$ & $\mathrm{C} 12 \mathrm{H} 16$ & 0.36 & 0.27 \\
\hline 162.995 & $\mathrm{C} 7 \mathrm{H} 3{ }^{37} \mathrm{ClF} 2 \mathrm{H}+$ & $\begin{array}{l}\text { parachlorobenzotrifluoride } \\
\text { (PCBTF) fragment }\end{array}$ & 0.00 & 0.07 \\
\hline 163.042 & $\mathrm{C} 9 \mathrm{H} 6 \mathrm{O} 3 \mathrm{H}+$ & $\begin{array}{c}\text { 1,3-isobenzofurandione, } 4- \\
\text { methyl- + isomers }\end{array}$ & 0.35 & 0.81 \\
\hline 163.074 & $\mathrm{C} 10 \mathrm{H} 10 \mathrm{O} 2 \mathrm{H}+$ & methyl-cinnamate + isomers & 0.66 & 0.58 \\
\hline 163.094 & $\mathrm{C} 7 \mathrm{H} 14 \mathrm{O} 4 \mathrm{H}+$ & C7H14O4 & 0.07 & 0.09 \\
\hline 164.095 & $\mathrm{C} 6 \mathrm{H} 13 \mathrm{NO} 4 \mathrm{H}+$ & $\mathrm{C} 6 \mathrm{H} 13 \mathrm{NO} 4$ & 0.17 & 0.20 \\
\hline 165.088 & $\mathrm{C} 10 \mathrm{H} 12 \mathrm{O} 2 \mathrm{H}+$ & eugenol + isoeugenol & 0.68 & 0.44 \\
\hline 165.162 & $\mathrm{C} 12 \mathrm{H} 20 \mathrm{H}+$ & $\mathrm{C} 12 \mathrm{H} 20$ & 0.08 & 0.19 \\
\hline 167.059 & $\mathrm{C} 10 \mathrm{H} 11 \mathrm{ClH}+$ & $\mathrm{C} 10 \mathrm{H} 11 \mathrm{Cl}$ & 0.12 & 0.16 \\
\hline 167.083 & $\mathrm{C} 13 \mathrm{H} 10 \mathrm{H}+$ & 9H-fluorene + isomers & 0.43 & 0.40 \\
\hline 167.104 & $\mathrm{C} 10 \mathrm{H} 14 \mathrm{O} 2 \mathrm{H}+$ & $\mathrm{C} 10 \mathrm{H} 14 \mathrm{O} 2$ & 0.57 & 0.48 \\
\hline 168.066 & $\mathrm{C} 8 \mathrm{H} 9 \mathrm{NO} 3 \mathrm{H}+$ & C8H9NO3 & 0.61 & 0.65 \\
\hline 169.062 & $\mathrm{C} 12 \mathrm{H} 8 \mathrm{OH}+$ & dibenzofuran & 0.73 & 0.62 \\
\hline 169.085 & $\mathrm{C} 9 \mathrm{H} 12 \mathrm{O} 3 \mathrm{H}+$ & $\mathrm{C} 9 \mathrm{H} 12 \mathrm{O} 3$ & 0.67 & 0.65 \\
\hline 169.119 & $\mathrm{C} 10 \mathrm{H} 16 \mathrm{O} 2 \mathrm{H}+$ & pinonaldehyde & 0.29 & 0.45 \\
\hline 170.932 & $\mathrm{C} 2 \mathrm{H} 3 \mathrm{IOH}+$ & acetyl iodide & 0.02 & 0.41 \\
\hline 171.17 & $\mathrm{C} 11 \mathrm{H} 22 \mathrm{OH}+$ & 2-undecanone + isomers & 0.23 & 0.45 \\
\hline 179.082 & $\mathrm{C} 14 \mathrm{H} 10 \mathrm{H}+$ & phenanthrene + isomers & 0.64 & 0.56 \\
\hline 179.178 & $\mathrm{C} 13 \mathrm{H} 22 \mathrm{H}+$ & $\mathrm{C} 13 \mathrm{H} 22$ & 0.08 & 0.18 \\
\hline 181.082 & $\mathrm{C} 10 \mathrm{H} 12 \mathrm{O} 3 \mathrm{H}+$ & $\mathrm{C} 10 \mathrm{H} 12 \mathrm{O} 3$ & 0.57 & 0.63 \\
\hline 183.092 & $\mathrm{C} 12 \mathrm{H} 10 \mathrm{~N} 2 \mathrm{H}+$ & $\mathrm{C} 12 \mathrm{H} 10 \mathrm{~N} 2$ & 0.58 & 0.58 \\
\hline 191.105 & $\mathrm{C} 12 \mathrm{H} 14 \mathrm{O} 2 \mathrm{H}+$ & $\mathrm{C} 12 \mathrm{H} 14 \mathrm{O} 2$ & 0.08 & 0.25 \\
\hline 191.178 & $\mathrm{C} 14 \mathrm{H} 22 \mathrm{H}+$ & $\mathrm{C} 14$ aromatics & 0.20 & 0.32 \\
\hline 193.194 & $\mathrm{C} 14 \mathrm{H} 24 \mathrm{H}+$ & $\mathrm{C} 14 \mathrm{H} 24$ & 0.04 & 0.18 \\
\hline 205.196 & $\mathrm{C} 15 \mathrm{H} 24 \mathrm{H}+$ & sesquiterpenes & 0.10 & 0.11 \\
\hline 207.207 & $\mathrm{C} 15 \mathrm{H} 26 \mathrm{H}+$ & $\mathrm{C} 15 \mathrm{H} 26$ & 0.05 & 0.20 \\
\hline
\end{tabular}




\begin{tabular}{|c|c|c|c|c|}
257.245 & $\mathrm{C} 16 \mathrm{H} 32 \mathrm{O} 2 \mathrm{H}+$ & $\mathrm{C} 16 \mathrm{H} 32 \mathrm{O} 2$ & 0.07 & 0.09 \\
\hline 297.083 & $\mathrm{C} 8 \mathrm{H} 24 \mathrm{O} 4 \mathrm{Si} 4 \mathrm{H}+$ & siloxane D4 & 0.00 & 0.02 \\
\hline 371.095 & $\mathrm{C} 10 \mathrm{H} 30 \mathrm{O} 5 \mathrm{Si} 5 \mathrm{H}+$ & siloxane D5 & 0.00 & 0.01 \\
\hline
\end{tabular}

\section{REFERENCES}

(1) Hynes, A. J.; Wine, P. H.; Nicovich, J. M. Kinetics and Mechanism of the Reaction of Hydroxyl Radicals with Acetonitrile under Atmospheric Conditions. J. Phys. Chem. 1988, 92 (13), 3846-3852. https://doi.org/10.1021/j100324a034.

(2) Kleinman, L. I.; Daum, P. H.; Lee, Y. N.; Nunnermacker, L. J.; Springston, S. R.; Weinstein-Loyd, J.; Hyde, P.; Doskey, P.; Rudolph, J.; Fast, J.; Berkowitz, C. Photochemical Age Determinations in the Phoenix Metropolitan Area. J. Geophys. Res. D Atmos. 2003, 108 (3). https://doi.org/10.1029/2002jd002621.

(3) Koss, A. R.; Sekimoto, K.; Gilman, J. B.; Selimovic, V.; Coggon, M. M.; Zarzana, K. J.; Yuan, B.; Lerner, B. M.; Brown, S. S.; Jimenez, J. L.; Krechmer, J.; Roberts, J. M.; Warneke, C.; Yokelson, R. J.; De Gouw, J. Non-Methane Organic Gas Emissions from Biomass Burning: Identification, Quantification, and Emission Factors from PTR-ToF during the FIREX 2016 Laboratory Experiment. Atmos. Chem. Phys. 2018, 18 (5), 32993319. https://doi.org/10.5194/acp-18-3299-2018.

(4) Coggon, M. M.; Veres, P. R.; Yuan, B.; Koss, A.; Warneke, C.; Gilman, J. B.; Lerner, B. M.; Peischl, J.; Aikin, K. C.; Stockwell, C. E.; Hatch, L. E.; Ryerson, T. B.; Roberts, J. M.; Yokelson, R. J.; de Gouw, J. A. Emissions of Nitrogen-Containing Organic Compounds from the Burning of Herbaceous and Arboraceous Biomass: Fuel Composition Dependence and the Variability of Commonly Used Nitrile Tracers. Geophys. Res. Lett. 2016, 43 (18), 9903-9912. https://doi.org/10.1002/2016GL070562.

(5) Huangfu, Y.; Yuan, B.; Wang, S.; Wu, C.; He, X.; Qi, J.; de Gouw, J.; Warneke, C.; Gilman, 
J. B.; Wisthaler, A.; Karl, T.; Graus, M.; Jobson, B. T.; Shao, M. Revisiting Acetonitrile as Tracer of Biomass Burning in Anthropogenic-Influenced Environments. Geophys. Res. Lett. 2021, 48 (11). https://doi.org/10.1029/2020GL092322.

(6) Stein, A. F.; Draxler, R. R.; Rolph, G. D.; Stunder, B. J. B.; Cohen, M. D.; Ngan, F. Noaa's Hysplit Atmospheric Transport and Dispersion Modeling System. Bull. Am. Meteorol. Soc. 2015, 96 (12), 2059-2077. https://doi.org/10.1175/BAMS-D-14-00110.1.

(7) Petit, J. E.; Favez, O.; Albinet, A.; Canonaco, F. A User-Friendly Tool for Comprehensive Evaluation of the Geographical Origins of Atmospheric Pollution: Wind and Trajectory $\begin{array}{lllll}\text { Analyses. Environ. } & \text { Model. Softw. }\end{array}$ https://doi.org/10.1016/j.envsoft.2016.11.022.

(8) Hsu, Y. K.; Holsen, T. M.; Hopke, P. K. Comparison of Hybrid Receptor Models to Locate PCB Sources in Chicago. Atmos. Environ. 2003, 37 (4), 545-562. https://doi.org/10.1016/S1352-2310(02)00886-5.

(9) Liang, Y.; Jen, C. N.; Weber, R. J.; Misztal, P. K.; Goldstein, A. H. Chemical Composition of PM2.5 in October 2017 Northern California Wildfire Plumes. Atmos. Chem. Phys. 2021, 21 (7), 5719-5737. https://doi.org/10.5194/acp-21-5719-2021.

(10) Knoderer, C.; Nguyen, D.; Alrick, D.; Hoag, K. 2016 Air Monitoring Network Plan; 2017.

(11) Long, R. W.; Whitehill, A.; Habel, A.; Urbanski, S.; Halliday, H.; Colón, M.; Kaushik, S.; Landis, M. S. Comparison of Ozone Measurement Methods in Biomass Burning Smoke: An Evaluation under Field and Laboratory Conditions. Atmos. Meas. Tech. 2021, 14 (3), 1783-1800. https://doi.org/10.5194/amt-14-1783-2021.

(12) Pfannerstill, E. Y.; Wang, N.; Edtbauer, A.; Bourtsoukidis, E.; Crowley, J. N.; Dienhart, D.; Eger, P. G.; Ernle, L.; Fischer, H.; Hottmann, B.; Paris, J.-D.; Stönner, C.; Tadic, I.; Walter, 
D.; Lelieveld, J.; Williams, J. Shipborne Measurements of Total OH Reactivity around the Arabian Peninsula and Its Role in Ozone Chemistry. Atmos. Chem. Phys. 2019, 19 (17), 11501-11523. https://doi.org/10.5194/acp-19-11501-2019.

(13) Misztal, P. K.; Lymperopoulou, D. S.; Adams, R. I.; Scott, R. A.; Lindow, S. E.; Bruns, T.; Taylor, J. W.; Uehling, J.; Bonito, G.; Vilgalys, R.; Goldstein, A. H. Emission Factors of Microbial Volatile Organic Compounds from Environmental Bacteria and Fungi. Environ. Sci. Technol. 2018, 52 (15), 8272-8282. https://doi.org/10.1021/acs.est.8b00806. 